\title{
Prognostizierte Veränderungen der gestaltbaren Arbeitssystemdimensionen
}

\author{
Steffen Wischmann und Ernst Andreas Hartmann
}

\subsection{Einleitung}

In den folgenden Kapiteln werden aktuelle Forschungs- und Entwicklungsprojekte vorgestellt, die exemplarisch aufzeigen, wie die Zukunft der Arbeit in Industrie 4.0 - bezogen auf ganz spezifische Anwendungsszenarien - aussehen könnte. Diese Beispiele erheben auch in ihrer Gesamtheit - keinen Anspruch auf Repräsentativität für die Arbeitswelt der Zukunft. Sie sollen vielmehr exemplarisch und prototypisch aufzeigen, welche prinzipiellen Ansatzpunkte und Dimensionen der Gestaltung zukünftiger Arbeitssysteme und -prozesse im Hinblick auf die Gestaltungspraxis von Bedeutung sind. Dadurch sollen zugleich grundlegende Konzepte der Gestaltung der Zukunft der Arbeit in Industrie 4.0 (z. B. Botthof und Hartmann 2015) konkretisiert und hinsichtlich ihrer praktischen Anwendbarkeit erprobt werden. Deswegen orientieren sich alle Beiträge an einem gemeinsamen Satz von Beschreibungs- und Gestaltungsdimensionen, die aus den genannten grundlegenden Konzepten abgeleitet sind.

In früheren Publikationen wurde besonders die Lernförderlichkeit von Arbeit als ein Leitmerkmal guter Arbeitsgestaltung herausgestellt (Hartmann 2005; Mühlbradt 2015; Hartmann und Garibaldo 2011; Henning et al. 1994). Dies lässt sich mehrfach begründen. Erstens ist angesichts des demografischen Wandels und längerer Erwerbsbiografien in Kombination mit beschleunigter technischer Innovation eine zunehmende Bedeutung des lebenslangen Lernens offensichtlich, wobei zugleich das informelle Lernen im

S. Wischmann $(\triangle) \cdot$ E. A. Hartmann

Institut für Innovation und Technik (iit) in der VDI/VDE Innovation + Technik GmbH, Steinplatz

1, 10623 Berlin, Deutschland

e-mail: wischmann@iit-berlin.de; hartmann@iit-berlin.de 
Arbeitsprozess - ergänzend zu formalem und nicht-formalem Lernen in Weiterbildungseinrichtungen - besondere Aufmerksamkeit verdient (Erpenbeck et al. 2007).

Zweitens sind die spezifischen Merkmale lernförderlicher Arbeit den grundlegenden Merkmalen guter, menschengerechter Arbeit sehr ähnlich (Hacker 2005; Hartmann et al. 2014).

Drittens besteht ein bedeutsamer Zusammenhang zwischen lernförderlicher Arbeit und Innovationen; lernförderliche Arbeit scheint in diesem Sinne ein wesentlicher Faktor der Innovationsfähigkeit von Unternehmen und Ländern zu sein (Lorenz 2015). Dies ist insofern auch plausibel, als einerseits Lernen und Innovation funktional nahe beieinander sind, andererseits Lernfähigkeit in allen Bereichen der Organisation, des Unternehmens wichtig ist, um neue Ideen von außen aufnehmen oder im Unternehmen selbst generieren und nachhaltig umzusetzen zu können.

Aus diesen Gründen werden viele der im Folgenden dargestellten Gestaltungsdimensionen einen Bezug zur lernförderlichen Arbeitsgestaltung aufweisen. Diese Dimensionen bilden zugleich die Grundlage für ein allgemeines arbeitsplatznahes Beschreibungsmodell der ,Arbeitswelt Industrie 4.0“ (Beitrag von Bauer, Schlund und Strölin in diesem Band).

In Anlehnung an die aktuelle Fachdiskussion (z. B. Hirsch-Kreinsen 2015; HirschKreinsen et al. 2015) ordnen wir für die Darstellung in diesem Kapitel die im Praxisteil dieses Buches - den folgenden Kapiteln - beschriebenen Tätigkeitsprofile zwei grundsätzlichen Kategorien ${ }^{1} \mathrm{zu}$ :

1. Operative Tätigkeiten: Hierzu zählen wir alle Tätigkeitsprofile, deren Arbeitsschwerpunkte auf ausführenden Tätigkeiten liegen, die zur Herstellung eines Produktes oder einer Dienstleistung notwendig sind. In diesem Segment handelt es sich um Tätigkeitsprofile, die i. d. R. von Arbeitnehmern mit niedrigem und mittlerem Qualifikationsniveau wahrgenommen werden.

- In diese Kategorie fallen insgesamt 24 der in den folgenden Kapiteln beschriebenen Tätigkeitsprofile, die sich in folgende Bereiche gruppieren lassen: Kommissionierung, Verpackung, Wareneingangskontrolle, gering qualifizierte Tätigkeiten, Fachkräfte, Applikateure, Maschinenmechaniker, Maschinenführer, Instandhaltung, Bediener, Monteure, Technischer Service, angelernte Montagemitarbeiter. ${ }^{2}$

2. Dispositive Tätigkeiten: Hierzu sollen alle Tätigkeitsprofile gehören, deren Arbeitsschwerpunkte auf der Planung, Organisation, Steuerung und Kontrolle liegen. In diesem Segment handelt es sich i. d. R. um Tätigkeitsprofile, die Arbeitnehmern mit gehobenem mittlerem und hohem Qualifikationsniveau zuzuordnen sind.

\footnotetext{
${ }^{1}$ Die Einteilung in diese beiden Kategorien erhebt keinen Anspruch auf Allgemeingültigkeit. Sie dient hier vielmehr dem praktischen Zweck, der Heterogenität der beschriebenen Tätigkeitsprofile gerecht zu werden und dabei dennoch konkrete qualitative Aussagen treffen zu können.

${ }^{2}$ Die Tätigkeitsbezeichnungen wurden unverändert aus den Anwendungsfallbeschreibungen übernommen.
} 
- In diese Kategorie fallen insgesamt 18 der beschriebenen Tätigkeitsprofile, die sich in folgende Bereiche gruppieren lassen: Leitungspersonen, Meister, Prozessplaner, Konstrukteur, Entwicklung, SPS-Programmierer, Verwaltung, Qualitätssicherung, Produktionsplaner, IT-Spezialisten.

Die Entwicklungsrichtung der Gestaltungsdimensionen wird im Folgenden anhand dieser Kategorien zusammenfassend über alle Praxisbeiträge analysiert und diskutiert. In den folgenden Abbildungen sind jeweils über die Praxisbeiträge hinweg akkumulierte Einschätzungen zur Entwicklungstendenz der jeweiligen Dimension dargestellt.

\subsection{Gestaltungsdimensionen}

\subsubsection{Bedarf}

Dieser erste Aspekt ist weniger eine Gestaltungs- als eine Wirkungsdimension. Die neu gestalteten Arbeitssysteme werden oftmals zu veränderten Bedarfen im Hinblick auf unterschiedliche Beschäftigtentypen oder Qualifikationsprofile (z. B. Konstrukteure, Einrichter, SPS-Programmierer) führen. Diese Entwicklungen können auch durchaus gegenläufig sein: höherer Bedarf nach dem einen, geringerer Bedarf nach dem anderen Beschäftigtentypus.

Es liegen übergreifende, quantitative Analysen von Arbeitsmarktdaten vor, die sich einerseits auf die Substituierbarkeit von bestehenden Tätigkeiten durch Technologie (Dengler und Matthes 2015; Frey und Osborne 2013) oder auch andererseits eher auf die Generierung neuer Tätigkeiten durch Technologie (Stewart et al. 2015) beziehen. In den folgenden Praxisbeiträgen wird demgegenüber auf sehr konkreter, exemplarischer Ebene abgeschätzt, welche Auswirkungen technisch-organisatorische Änderungen auf die Nachfrage nach bestimmten Beschäftigtentypen in bestimmten Anwendungsszenarien haben können.

Über alle Tätigkeitsprofile hinweg gehen die Autoren der folgenden Beiträge deutlich öfter von einem Mehr- als von einem Minderbedarf an Arbeitskräften aus (Abb. 2.1 links). Dies gilt sowohl für operative als auch dispositive Tätigkeiten (Abb. 2.1 Mitte, rechts). In der Hälfte aller beschriebenen Tätigkeiten werden keine Veränderungen erwartet.

\subsubsection{Hierarchische Vollständigkeit}

Hacker unterscheidet zwischen einem hierarchischen und einem sequenziellen Aspekt der Vollständigkeit von Tätigkeiten (Hacker 2005). Die hierarchische Vollständigkeit bezieht sich auf die Mischung von weniger anspruchsvollen Tätigkeiten - z. B. Routinetätigkeiten, Tätigkeiten, die nach klaren und einfachen Regeln zu bewältigen sind - und anspruchsvollen Tätigkeiten, z. B. schöpferische und problemlösende Tätigkeiten. Wenn 


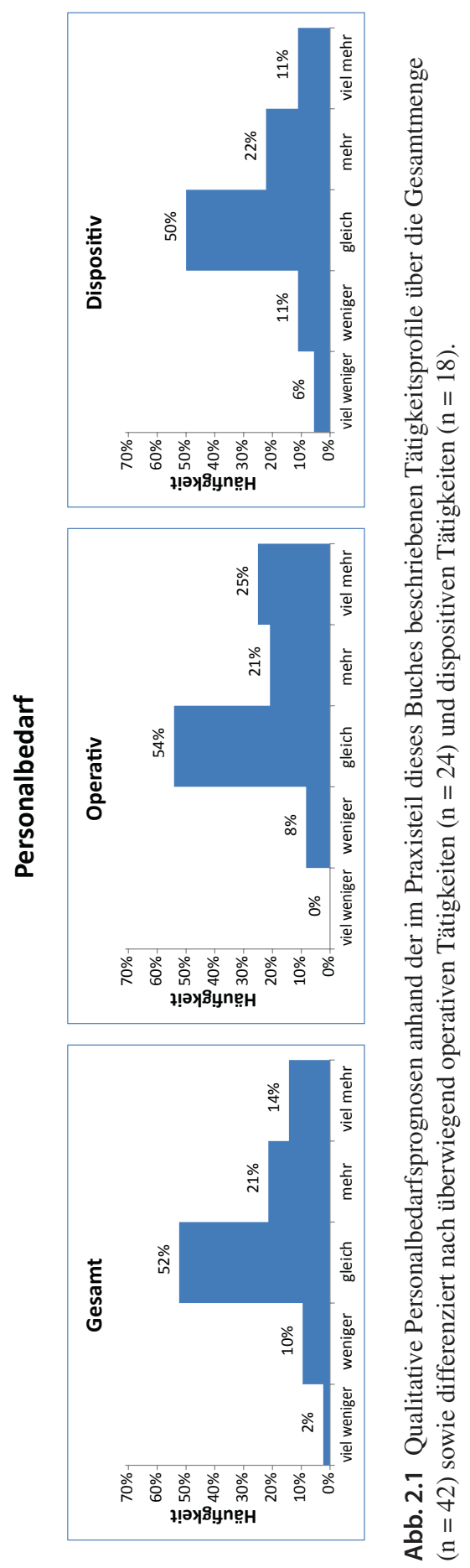


im Aufgabenprofil eines Mitarbeiters (an einem Arbeitsplatz) ein ausgewogenes Verhältnis von anspruchsvollen und weniger anspruchsvollen Teiltätigkeiten besteht, ist insgesamt die Tätigkeit hierarchisch vollständig.

Das Anforderungsniveau dieser Tätigkeiten ist dabei immer im Hinblick auf ganz bestimmte Individuen oder Typen von Individuen zu beurteilen: Je nach persönlichen Merkmalen (z. B. Vorbildung, körperliche und geistige Leistungsfähigkeit) sind die gleichen Aufgaben für unterschiedliche Personen unterschiedlich anspruchsvoll (interindividuelle Unterschiede, differentieller Aspekt (Ulich 1978; 2016)).

Die hierarchische Vollständigkeit ist zugleich einem kontinuierlichen Wandel unterworfen: Durch Lernprozesse werden etwa vormals sehr anspruchsvolle Tätigkeiten für die gleichen Individuen über die Zeit weniger anspruchsvoll ${ }^{3}$ (intraindividuelle Unterschiede, dynamischer Aspekt (Ulich 1978; 2016)). Aus der Sicht der praktischen Arbeitssystemgestaltung ist deshalb das Konzept der evolvierend vollständigen Tätigkeit bedeutsam: Um über die Zeit hinweg hierarchisch vollständig zu bleiben, muss eine Tätigkeitsstruktur mit dem Kompetenzzuwachs des Individuums „mitwachsen“ (Hacker und Richter 1990).

Vor diesem Hintergrund werden in den folgenden Abschnitten im Kontext der hierarchischen Vollständigkeit folgende Aspekte angesprochen:

- Monotone und komplexe Aufgaben

- Problemlösung und Optimierung

- Lernen

\subsubsection{Monotone und komplexe Aufgaben}

Monotonie als psychischer Zustand wird hervorgerufen durch reizarme Situationen und repetitive Tätigkeiten. Unmittelbare Symptome sind Müdigkeit, Interessenlosigkeit und Gefühle der Langeweile. Zwischen dem Anteil monotoner Aufgaben und der Lernförderlichkeit der Arbeitsaufgaben insgesamt besteht ein negativer Zusammenhang (Bergmann et al. 2000; Bergmann et al. 2004).

Zugleich stellen monotone Aufgaben auch grundsätzlich eine Fehlbeanspruchung dar, die zu Leistungsminderung, Leistungsschwankungen und sinkender Lernfähigkeit sowie -bereitschaft führt (Ulich 2005; Richter und Hacker 1997). Besonders kritisch sind monotone Aufgaben, die zugleich hohe und kontinuierliche Aufmerksamkeit erfordern (z. B. Überwachungstätigkeiten) (Bainbridge 1983). In solchen Fällen ist es nicht möglich, den Monotoniezustand durch Aufgabenwechsel zu beenden bzw. zu unterbrechen.

Ein hoher Anteil monotoner Aufgaben beeinträchtigt die hierarchische Vollständigkeit von Tätigkeiten. Ansatzpunkte für die Gestaltung liegen hier im Bereich der Technik bis hin zur vollständigen Automatisierung monotoner Tätigkeiten - und im Bereich der Arbeitsorganisation mit dem Ziel, monotone und anspruchsvolle Aufgaben an Arbeitsplätzen zu kombinieren.

\footnotetext{
${ }^{3}$ Umgekehrt kann der subjektive Anspruchsgehalt von Aufgaben durch Verluste in der individuellen Leistungsfähigkeit auch steigen.
} 
Komplexe Aufgaben können als Gegenpol zu den monotonen Aufgaben betrachtet werden, sie stellen ein wesentliches Merkmal lernförderlicher Arbeitsplätze dar (Bergmann et al. 2004; Frieling et al. 2006; Bergmann et al. 2000). Komplexe Aufgaben erfordern ein hohes Kompetenzniveau der Beschäftigten und ermöglichen zugleich die fortlaufende Kompetenzentwicklung in der Arbeit.

Auch die Innovationsfähigkeit von Unternehmen und Ländern scheint in besonderer Weise vom Anteil komplexer Aufgaben abzuhängen (Lorenz und Valeyre 2005; Lorenz 2015; Hartmann et al. 2014). Dieser Zusammenhang stellt sich über zwei Mechanismen her. Einerseits gehen mit komplexen Aufgaben, wie oben bereits angesprochen, auch höhere Kompetenzniveaus der Beschäftigten einher. Dies erleichtert sowohl die Wahrnehmung externer Innovationsimpulse (z. B. neue Bearbeitungsverfahren) wie auch die interne Verarbeitung, Umsetzung und Verbreitung dieser Innovationsimpulse (Cohen und Levinthal 1990). Andererseits erhöht die höhere Lernfähigkeit auch die Wahrscheinlichkeit, dass intern (z. B. im Zuge kontinuierlicher Verbesserungsprozesse) Innovationen erdacht und umgesetzt werden können.

Der Anteil komplexer Aufgaben ist, wie oben angesprochen, auch eng verbunden mit dem arbeitspsychologischen Konzept der hierarchisch vollständigen Tätigkeit (Hacker 1995, 2005). Hierarchische Vollständigkeit bedeutet, dass Aufgaben unterschiedlichen Anforderungsniveaus in einem angemessenen Mischungsverhältnis an einem Arbeitsplatz vorhanden sein sollten. In diesem Sinne ist auch ein gewisser Anteil anforderungsarmer oder sogar monotoner Aufgaben hinnehmbar, wenn auch hinreichend viele komplexe Aufgaben zum Portfolio eines Arbeitsplatzes gehören.

Aus den Praxisbeiträgen wird eine klare Tendenz deutlich. Sowohl auf operativer als auch auf dispositiver Ebene wird mit einer deutlichen Abnahme von monotonen Aufgabenanteilen bei gleichzeitiger starker Zunahme von komplexen Aufgabenanteilen ausgegangen (siehe Abb. 2.2). Aus Sicht der oben diskutierten Innovationsfähigkeit handelt es sich dabei sicherlich um eine sehr positive Entwicklungstendenz. Auch im Hinblick auf gut gestaltete Arbeit ist eine solche Entwicklung wünschenswert, weil durch die tendenziell steigende Komplexität der Aufgaben auch bei kontinuierlichen Kompetenzgewinnen der Individuen hierarchisch vollständige Tätigkeiten - und insbesondere evolvierend vollständige Tätigkeiten - entstehen bzw. erhalten bleiben (Hacker und Richter 1990).

\subsubsection{Problemlösung und Optimierung}

Problemlösende Tätigkeiten haben eine besonders enge Beziehung zur Lernförderlichkeit des Arbeitsplatzes; je höher der Anteil problemlösender Aufgaben, desto höher auch die Lernchancen (Hacker 2005; Pietzcker 2010). Es ist daher ein wichtiges Ziel der Arbeitsorganisation, Arbeitsaufgaben so zu gestalten und an Arbeitsplätzen zu kombinieren, dass zumindest ein Minimum problemlösender Tätigkeiten möglichst überall vorhanden ist.

Der Anforderungsgehalt problemlösender Tätigkeiten kann weiter danach unterschieden werden, inwieweit der Ist-Zustand, der Soll-Zustand und die Operatoren, die den Istin den Soll-Zustand überführen können, dem Problemlöser bekannt sind. Sind alle diese Aspekte bekannt, handelt es sich nicht um Probleme, sondern um nach bekannten Regeln zu bearbeitende Aufgaben (Sell und Schimweg 2002). 

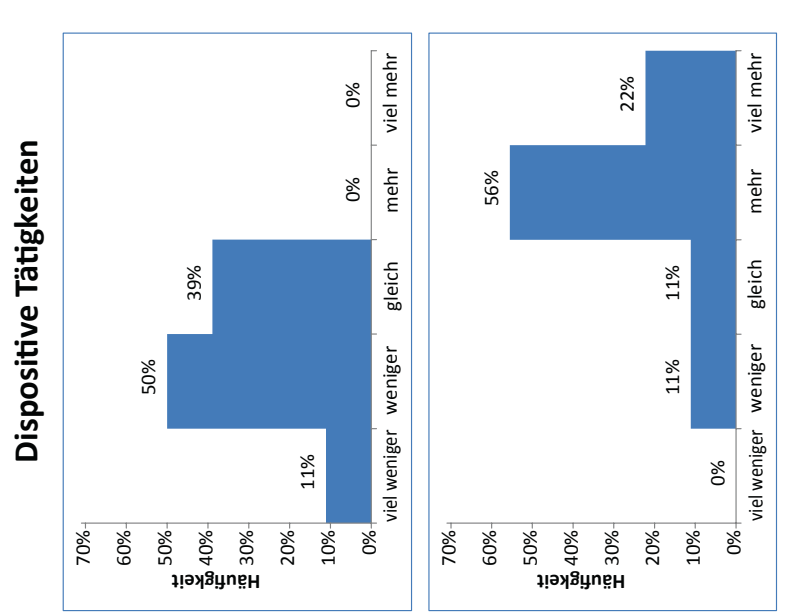

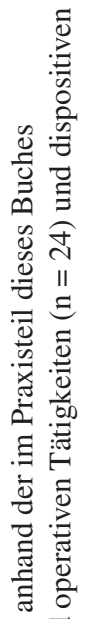
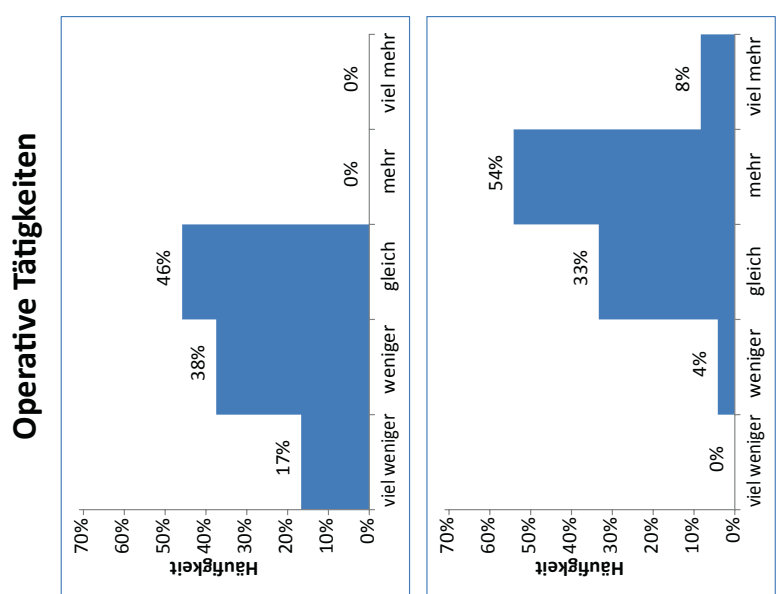

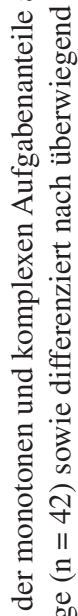

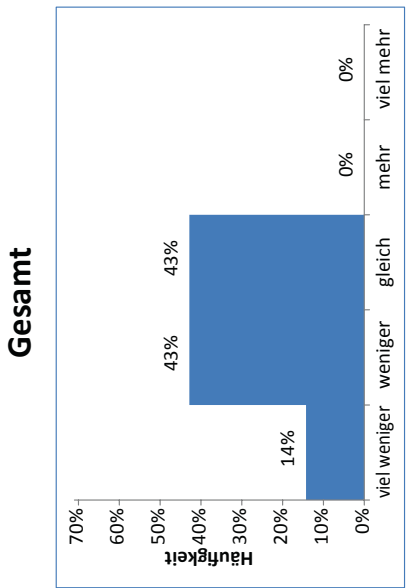

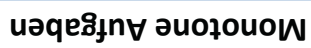

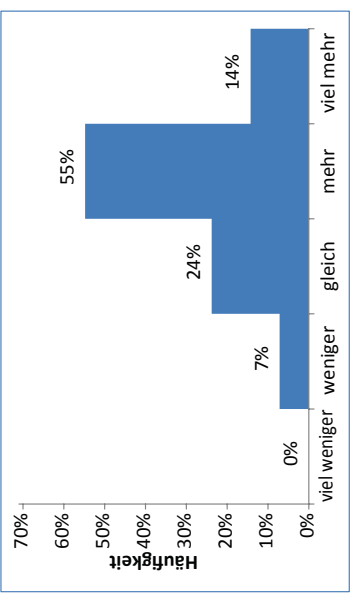

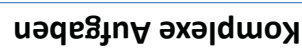

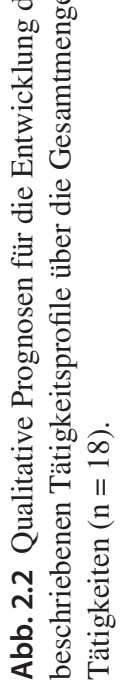


Problemlösende Aufgaben sind grundsätzlich eher komplex, in Abhängigkeit vom konkreten Anforderungsgehalt der gestellten Probleme (vgl. vorigen Abschnitt). Zugleich sind problemlösende Aufgaben traditionell solche, die eher Menschen als Maschinen zugewiesen werden. Dies könnte sich allerdings durch steigende Leistungsfähigkeit künstlicher Intelligenz ändern. Gerade deshalb sollten aber die technischen und arbeitsorganisatorischen Möglichkeiten ausgenutzt werden, die dazu beitragen, einen hinreichenden Anteil problemlösender Tätigkeiten für die Menschen zu schaffen und zu erhalten.

Optimierungsaufgaben beziehen sich etwa auf die kontinuierliche Verbesserung von Prozessen im Arbeitskontext, auf Qualitäts- und Effektivitätssteigerungen. Optimierungsaufgaben haben eine große praktische Bedeutung, auch für die Anreicherung industrieller Arbeitstätigkeiten in Richtung auf eine lernförderliche Arbeitsgestaltung. Strukturell und auf die jeweiligen kognitiven Anforderungen bezogen sind Optimierungen Spezialfälle von Problemlöseprozessen (Kauffeld 2007).

Aus den Praxisbeiträgen ergibt sich ein gemischtes Bild. Sowohl auf operativer als auch auf dispositiver Ebene wird einerseits bei ungefähr der Hälfte aller beschriebenen Tätigkeitsprofile mit einer Zunahme an problemlösenden Aufgaben gerechnet (Abb. 2.3, oben). Das ist auf Grund der prognostizierten, oben diskutierten steigenden Aufgabenkomplexität durchaus erwartbar. Allerdings nimmt der Anteil an problemlösenden Aufgaben nach dieser Prognose auch bei rund einem Drittel aller beschriebenen Tätigkeiten ab (bei $38 \%$ der operativen und $28 \%$ der dispositiven Tätigkeiten). Hier spielt sicherlich die steigende Intelligenz der neuen Technologien eine entscheidende Rolle, die zunehmen in der Lage sein wird, Probleme zu lösen, die bislang nur durch menschliches Denken gelöst werden konnten.

Hinsichtlich der Optimierungsmöglichkeiten der Arbeitsprozesse und der Effizienz wird von einer deutlichen Zunahme ausgegangen, interessanterweise besonders stark bei den operativen Tätigkeiten (Abb. 2.3, unten). Hier zeigen sich Potenziale der Aufwertung von operativen Tätigkeiten.

\subsubsection{Lernen}

Grundsätzlich soll unterschieden werden zwischen informellem und formellem Lernen. ${ }^{4}$ Informelles Lernen tritt als ungeplanter und oftmals auch unintendierter „Nebeneffekt“ vielfältiger Tätigkeiten - Arbeit, soziale Engagement, Spiel - auf. Formelles Lernen hingegen findet in explizit dafür vorgesehenen Settings (z. B. Seminarraum) als geplanter und gezielt auf das Lernen hin gestalteter Prozess statt.

\footnotetext{
${ }^{4}$ Oftmals wird zwischen informellem, non-formalem und formalem Lernen unterschieden. Im Unterschied zu informellem Lernen findet non-formales Lernen nicht „nebenbei“ statt, sondern in expliziten Lernprozessen und -umgebungen; im Unterschied zum formalen Lernen werden keine allgemein akzeptierten Zertifikate (z. B. Abiturzeugnis, Hochschulabschluss) vergeben. Im Kontext dieses Kapitels werden das non-formale und das formale Lernen - gemäß ihrer gemeinsamen Merkmale der bewusst und gezielt gestalteten Lernumgebungen und -prozesse - unter dem Begriff des formellen Lernens subsummiert und vom informellen Lernen abgegrenzt.
} 

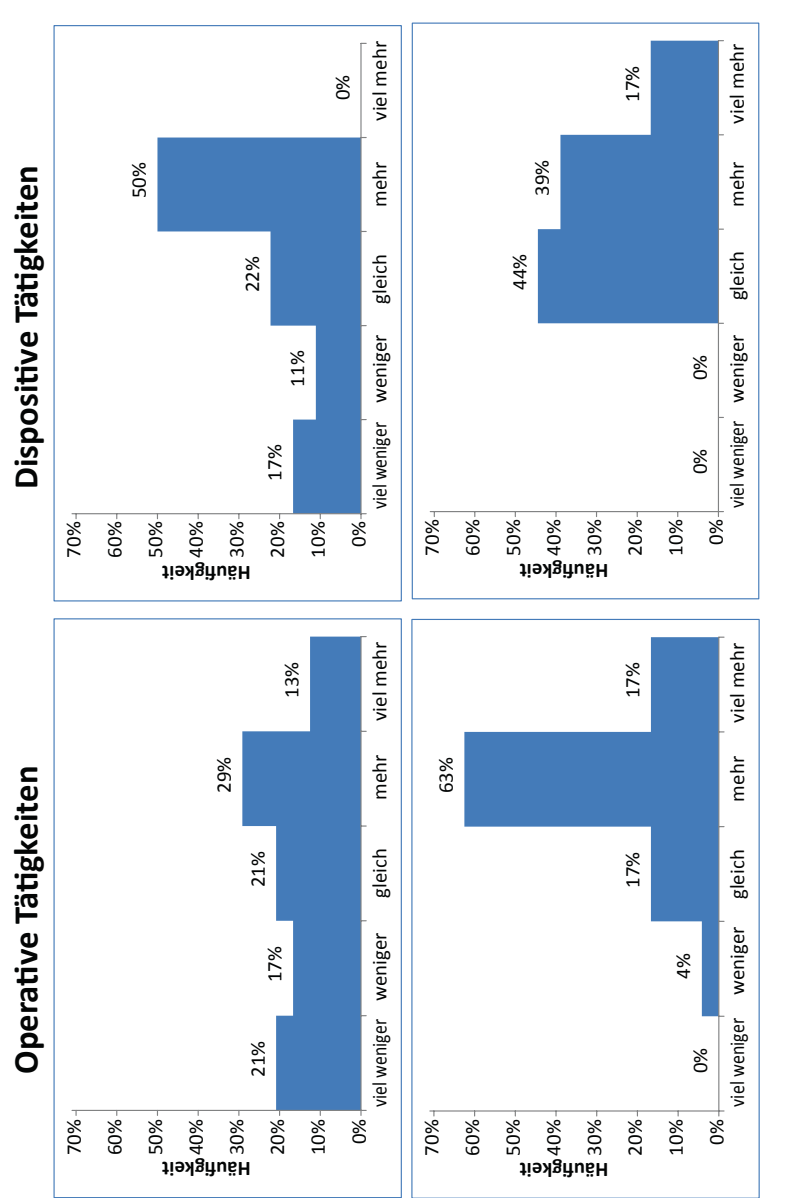

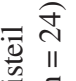

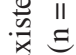

ล ำ

$\Xi \cdot \frac{\vec{v}}{\mathrm{~g}}$

宕:

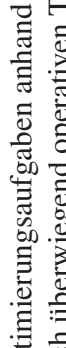

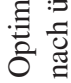

竞

를

ब्ञ

啳

.

해

蒘

ฮี II

$\Xi$

:0 80

है

ธิ

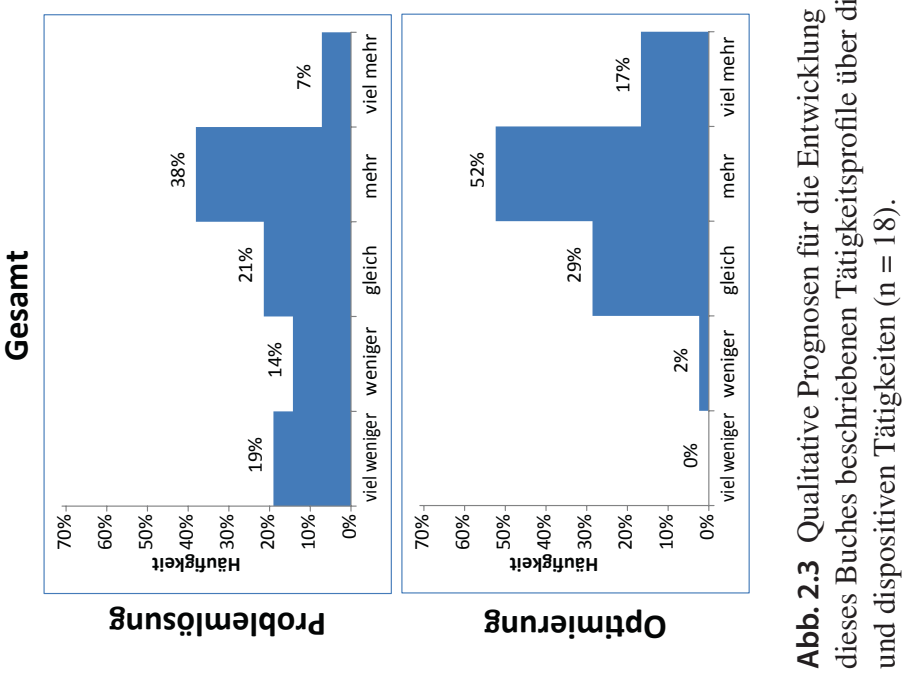


Aus einer arbeitswissenschaftlichen Perspektive ist informelles Lernen eine unmittelbare Folge lernförderlicher Arbeitsorganisation (Bergmann et al. 2004; Frieling et al. 2006). Aus einer bildungspolitischen Perspektive gewinnt gegenüber dem formellen Lernen (s. u.) das informelle Lernen außerhalb von Bildungseinrichtungen als wesentliches Element des lebenslangen Lernens an Bedeutung (Erpenbeck et al. 2007). Aus einer innovationsbezogenen Perspektive schließlich ist die Intensität des Lernens in der Arbeit - gemeinsam mit Kontrolle und Selbstbestimmung - ein zentrales Merkmal innovationsförderlicher Arbeitsgestaltung (OECD 2010; Lorenz 2015; Lorenz und Valeyre 2005).

Der relative Bedeutungsgewinn des informellen Lernens führt nicht zu einem grundlegenden Bedeutungsverlust des formellen Lernens. Im Gegenteil, es sind sowohl berufliche wie hochschulische Bildungsangebote in neuen Formaten und mit neuen Inhalten notwendig, um die mit dem technologischen Wandel einhergehenden Qualifikationsbedarfe zu decken. Dabei wird - vor dem Hintergrund des demografischen und technologischen Wandels - die Fort-und Weiterbildung gegenüber der initialen (Berufs-)Bildung an Bedeutung gewinnen.

Wie genau sich die Qualifikationsbedarfe entwickeln werden, hängt - auch bei gleichen Berufsgruppen und Anwendungsfeldern - sehr stark von der organisatorisch-technischen Gestaltung der jeweiligen Arbeitssysteme und -umgebungen ab. Es sind hochautomatisierte, tayloristische Gestaltungsszenarien denkbar, die quantitativ eher zu einem geringeren Bedarf an Arbeitskräften und qualitativ zu einer Polarisierung innerhalb der Beschäftigten führen könnten: Wenige Hochqualifizierte würden in einem solchen Szenario relativ vielen Geringqualifizierten gegenüberstehen.

Andererseits sind ebenso für fast alle Berufsgruppen und Anwendungsfälle Organisationsszenarien denkbar, die sehr viel stärker auf eine Nutzung, Unterstützung und kontinuierliche Erweiterung menschlicher Kompetenz - auch und gerade mit technischer Unterstützung (tutorielle Assistenzsysteme) - setzen. Hier würde kaum eine Tendenz zur Polarisierung auftreten und auch insgesamt wären die Qualifikationsanforderung andere (Hartmann und Bovenschulte 2013). In diesem Sinne ist das Maß an notwendiger Qualifikation eine abhängige Variable, die von der Ausprägung von Gestaltungsdimensionen wie Problemlösung, komplexe Aufgaben, Planen und Kontrolle abhängt.

Schließlich zeigen sich Tendenzen dahingehend, dass formelles und informelles Lernen auch stärker integrativ verstanden werden kann, etwa durch projektbasierte Bildungsangebote.

Gemäß der Prognose unserer Autoren werden sowohl formelles wie informelles Lernen deutlich an Bedeutung gewinnen, sowohl auf operativer als auch auf dispositiver Ebene (Abb. 2.4). Insbesondere das informelle Lernen wird mittels der neuen digitalen Technologien eine ganz zentrale Rolle spielen.

Das formelle Lernen wird es jedoch nicht ersetzen. Auf operativer Ebene wird erwartet, dass bei weit über der Hälfte der beschriebenen Tätigkeitsprofile ein zusätzlicher Bedarf besteht, neue Qualifikationen beispielsweise im Rahmen von Weiterbildungsmaßnahmen zu erwerben. Interessant ist, dass dies sogar noch stärker auf der dispositiven Ebene erwartet wird. Bei knapp $75 \%$ der beschriebenen Tätigkeitsprofile wird ein Anstieg der Anteile 


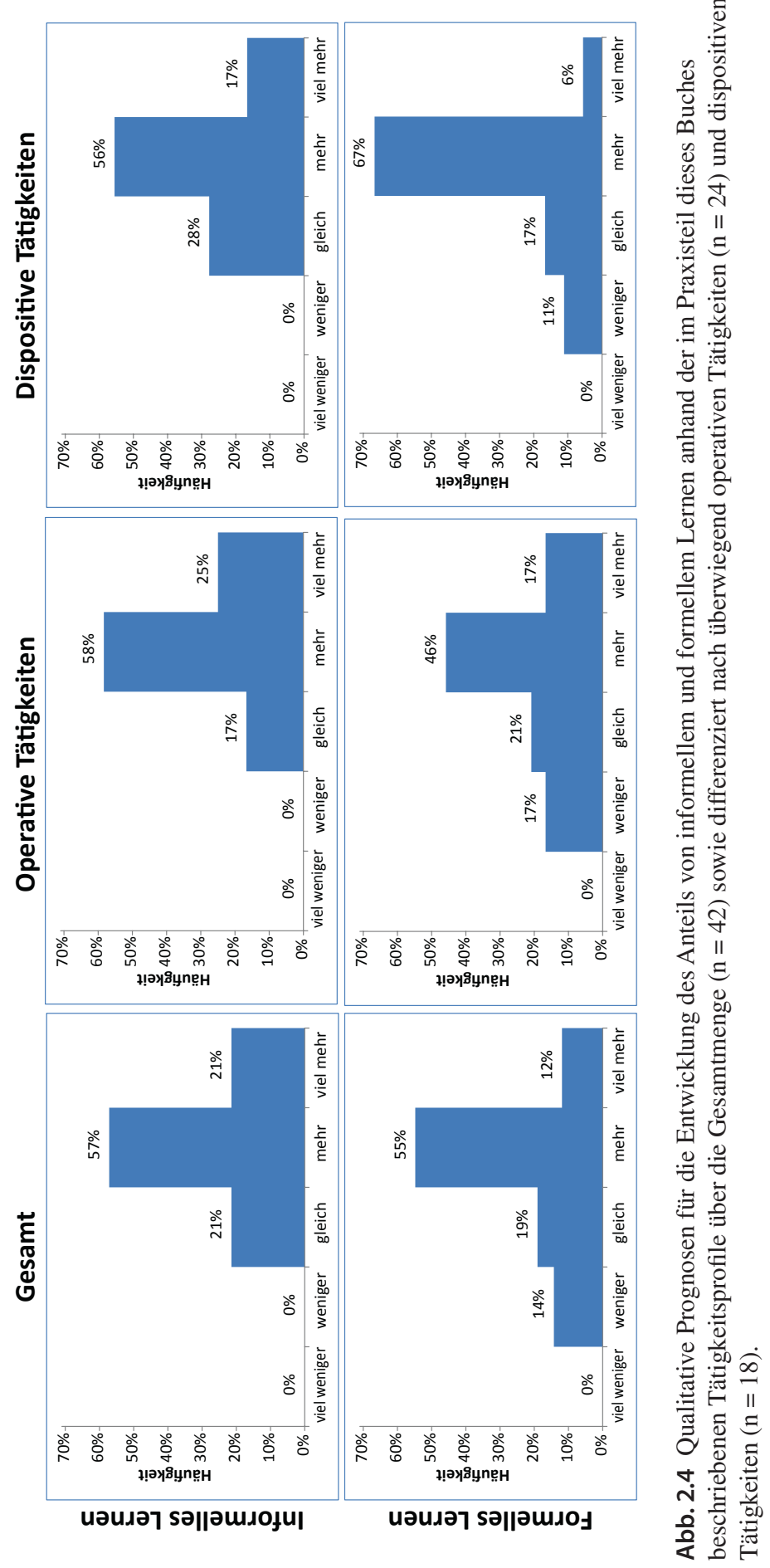


des formellen Lernens erwartet. Dies überrascht insoweit, als das es sich bei den betroffenen Mitarbeitern in der Regel um bereits hochqualifizierte Mitarbeiter handelt.

\subsubsection{Sequentielle Vollständigkeit von Tätigkeiten}

Wie oben bereits angesprochen, unterscheidet Hacker zwischen einem hierarchischen und einem sequenziellen Aspekt der Vollständigkeit von Tätigkeiten (Hacker 2005). Während sich die hierarchische Vollständigkeit auf die Mischung von mehr und weniger anspruchsvollen Tätigkeiten bezieht, orientiert sich die sequentielle Vollständigkeit an der Handlungssequenz (bzw. dem Handlungszyklus) bestehend aus:

- Organisieren im Sinne der Abstimmung der eigenen Tätigkeit mit den Tätigkeiten anderer Personen

- Planen der eigenen Tätigkeit

- Ausführen der Tätigkeit

- Kontrollieren der Ergebnisse der eigenen Tätigkeit

Im Folgenden wird zunächst - wegen der besonderen Bedeutung für die Arbeitsgestaltung - auf planerische Tätigkeitsanteile eingegangen. Daran schließt sich eine Diskussion von Kooperation und Kommunikation als Aspekte der organisierenden Abstimmung mit anderen an. Der Aspekt der Kontrolle wird in einem etwas weiter gefassten Sinn, der über die bloße Kontrolle eigener Arbeitsergebnisse hinausgeht, weiter unten (Abschn. 2.2.4) angesprochen.

\subsubsection{Planen}

Während monotone, problemlösende und komplexe Aufgaben in besonderer Weise mit dem Konzept der hierarchischen Vollständigkeit verbunden sind, hat der Anteil planender Aufgaben eine hohe Bedeutung für die sequentielle Vollständigkeit von Tätigkeiten (Hacker 1995, 2005). Sequentielle Vollständigkeit ist dann gegeben, wenn planende, organisierende, ausführende und kontrollierende Tätigkeiten an einem Arbeitsplatz vereint sind.

Die Automatisierung ausführender Tätigkeiten hat in der Vergangenheit oftmals zu einem relativen Bedeutungsgewinn planender Tätigkeiten für die Beschäftigten geführt. Dies könnte sich allerdings durch steigende Leistungsfähigkeit künstlicher Intelligenz insofern ändern, als dann auch zunehmend planende Aufgaben von technischen Systemen übernommen werden können.

Umso wichtiger ist es, die organisatorischen und technischen Möglichkeiten auszunutzen, um einen hinreichenden planerischen Aufgabenanteil an möglichst allen Arbeitsplätzen zu sichern.

Die Autoren der Anwendungsfälle erwarten über alle Tätigkeitsprofile hinweg keine Veränderung im Hinblick auf planerische Tätigkeitsanteile (Abb. 2.5). Interessant ist der 


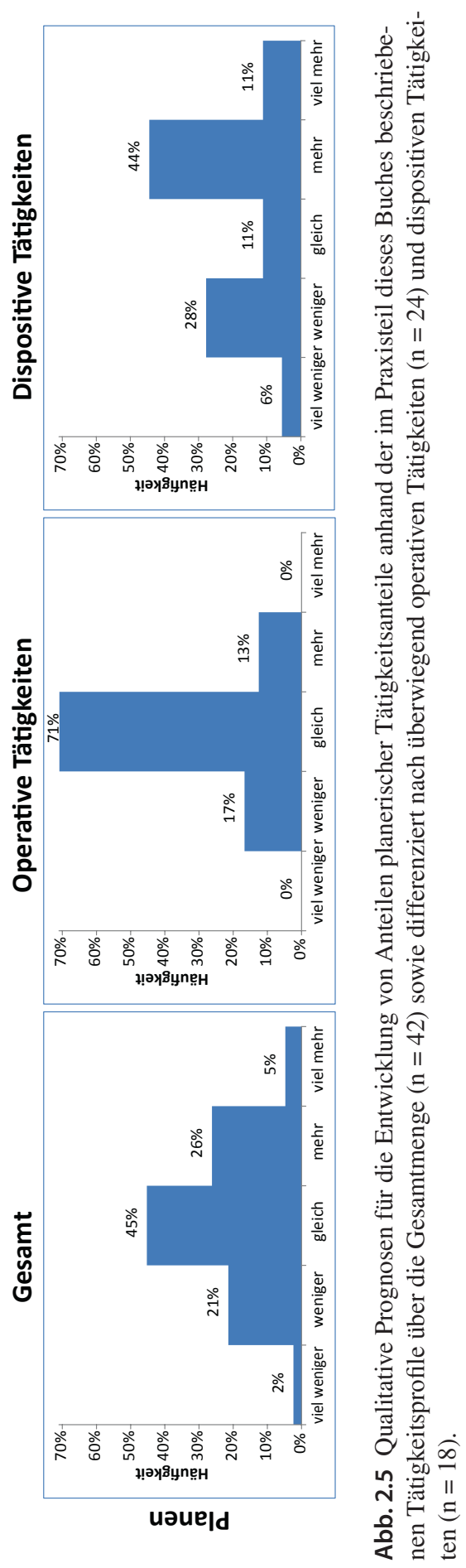


Vergleich zwischen operativen und dispositiven Tätigkeiten: Während bei den operativen Tätigkeiten im Wesentlichen keine Veränderungen erwartet werden, halten sich bei den dispositiven Tätigkeiten Ab- und Zunahme planerischer Tätigkeitsanteile ungefähr die Waage. Es zeigt sich hier erneut die Ambivalenz „smarter“" Systeme, die menschliche Planung sowohl unterstützen wie auch bis zur Überflüssigkeit vereinfachen und automatisieren können.

\subsubsection{Kooperation und Kommunikation}

Ein Aspekt sequentieller Vollständigkeit ist das Planen der eigenen Arbeitstätigkeit (s. o.), ein anderer ist das Organisieren, die Abstimmung des eigenen Handelns mit dem Handeln Anderer. Auch generell kann man die Möglichkeit, in der Arbeit mit anderen zu kooperieren, als ein Merkmal menschengerechter Arbeitsgestaltung begreifen (Zölch et al. 1999).

An dieser Stelle können durchaus Zielkonflikte der Arbeitssystemgestaltung auftreten. Je autonomer (s. u., Abschn. 2.2.4) etwa eine Individuum oder eine Gruppe in ihrer Arbeitstätigkeit ist, desto geringer können Kooperationsanforderungen und -notwendigkeiten in Bezug auf Andere ausfallen.

In ähnlicher Weise wie bei der Kooperation kann auch die Kommunikation - in Sinne von Gelegenheit oder auch Notwendigkeit der Kommunikation mit Anderen - als ein Merkmal sui generis menschengerechter Arbeitsgestaltung verstanden werden (Oesterreich und Dunckel 1993). Auch mögliche Zielkonflikte stellen sich ähnlich dar, wie oben im Falle der Kooperation beschrieben.

Hinsichtlich der Aspekte Kommunikation und Kooperation zeigt sich ein einheitliches Bild über alle im Praxisteil betrachteten Tätigkeitsprofile (Abb. 2.6). Beachtlich ist, dass in fast keinem diskutierten Arbeitsfeld mit einem Rückgang an kooperativen und kommunikativen Arbeitsanteilen gerechnet wird. Im Gegenteil - in ca. 70 \% aller Tätigkeitsprofile sowohl auf operativer als auch auf dispositiver Ebene wird mit einer deutlichen Zunahme beider Aspekte menschengerechter Arbeit gerechnet. Dies stellt allerdings auch neue Herausforderungen an die kommunikativen und kooperativen Fähigkeiten der Mitarbeiter.

\subsubsection{Kontrolle und Autonomie}

Die bislang unter den Kategorien der hierarchischen und sequentiellen Vollständigkeit diskutierten Gestaltungsdimensionen betonen eher kognitive Aspekte der Arbeitstätigkeit. Im Unterschied dazu stehen im Folgenden motivationale Faktoren im Vordergrund: Sowohl das Streben des Menschen nach Kontrolle über seine Umweltbedingungen als auch das eng damit zusammenhängende Streben nach Selbstbestimmung kennzeichnen zentrale menschliche Motive. Kontrolle im Sinne der Kontrolle des Menschen über seine Umweltbedingungen gehört zu den wichtigsten Gestaltungsaspekten menschlicher Arbeit (Grote 1997). Wichtige verwandte Konzepte sind Handlungs- und Entscheidungsspielräume 

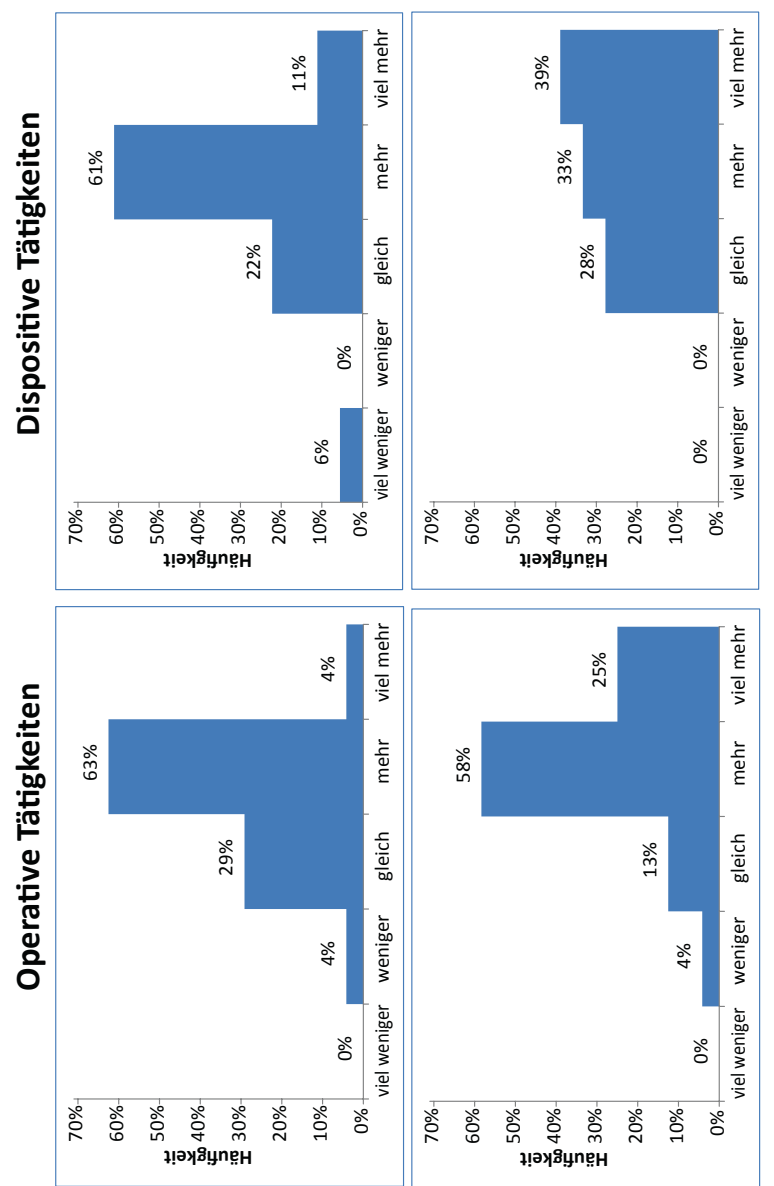

बे 吾: 岂

웡

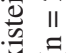

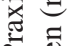

$\stackrel{2}{ \pm}$

$\Xi \frac{0}{0}$

牙

클

כ

항

馬

:

文

ขี :

氙

竝

壱.

을

롱

I.

.

สิ

응

t)

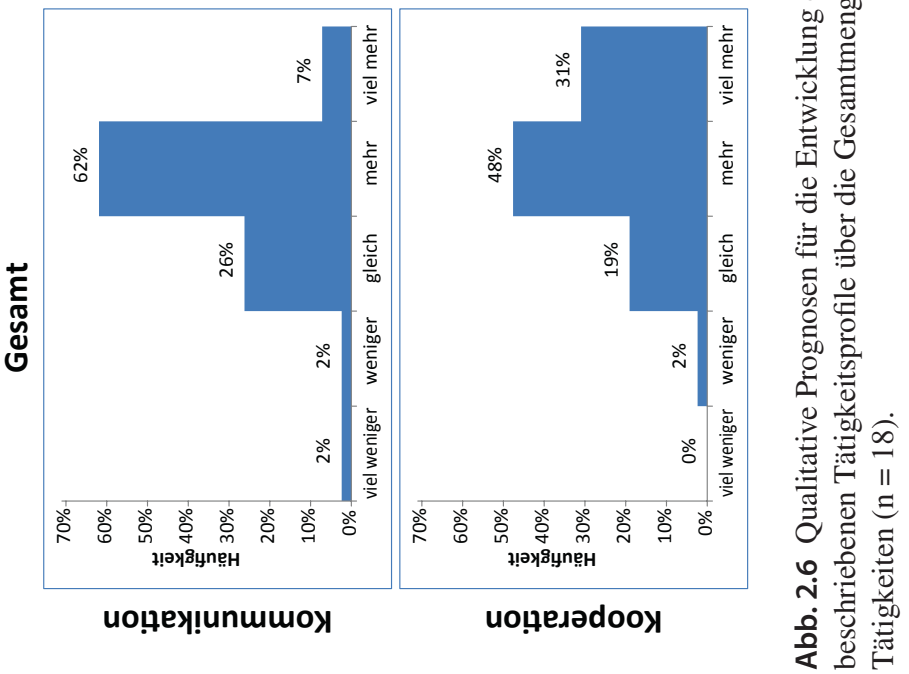


sowie Freiheitsgrade in der Arbeit (Hacker 2005). Für eine nachhaltige Sicherung der psychischen, aber auch der physischen Gesundheit ist eine Arbeitsgestaltung wichtig, welche die Beeinflussung der objektiven Arbeitsbedingungen durch die Arbeitenden ermöglicht. Ist dies nicht der Fall, wird von den Beschäftigten subjektiv keine Kontrolle über die Umwelt erlebt und es stellen sich Phänomene wir die erlernte Hilflosigkeit ein (Seligman 1995).

Eng verbunden mit Fragen der Kontrolle sind Automatisierungsdilemmata (Bainbridge 1983). In automatisierten Systemen übernehmen Menschen oftmals Überwachungstätigkeiten. Unkritische Situationen werden in der Regel vom automatischen System beherrscht. Situationen, in denen der überwachende Mensch eingreifen muss, sind tendenziell eher komplexe Situationen. Daraus entstehen zwei Dilemmata.

Erstens ist der Mensch in dieser Situation schlecht vorbereitet, die Kontrolle zu übernehmen, weil er die Situation nicht selbst herbeigeführt hat. Dadurch ist er weniger „im Bilde“. Dieser Effekt betrifft das Kurzzeit- bzw. Arbeitsgedächtnis.

Zweitens stellt sich über die Zeit auch eine durch mangelnde Übung versursachte Rückentwicklung der Fähigkeiten ein, die zur aktiven Bewältigung der Situation notwendig wären (skill erosion). Dieser Effekt betrifft das Langzeitgedächtnis.

Im Endeffekt wird der Mensch tendenziell mit besonders anspruchsvollen Situationen konfrontiert, zu deren Bewältigung er - automatisierungsbedingt - aus den zwei genannten Gründen weniger fähig ist. Hier besteht eine besondere Herausforderung an die Technikgestaltung. Es gilt, Mensch-Maschine-Schnittstellen zu entwerfen, die es den Menschen auch in hochautomatisierten Systemen erlauben, ,im Bilde“ zu sein und Kontrolle auszuüben; hierfür stehen auch geeignete Methoden zur Verfügung (Lüdtke 2015).

Der Aspekt der Selbstbestimmung (Autonomie) hängt eng mit dem oben angesprochenen Konstrukt der Kontrolle zusammen, geht aber weiter. So hat das Konzept der Selbstbestimmung eine deutliche normative, wertende Bedeutung, die tief im kulturellen Gewebe moderner, demokratischer, offener Bürgergesellschaften verankert ist. Weiterhin wird das Konzept der Selbstbestimmung oder Autonomie auch oftmals auf die kollektive Handlungsorganisation in Gruppen angewandt, man spricht dann auch von (teil-)autonomen Arbeitsgruppen (Weber 1997).

Die Autoren der Praxisfallbeschreibungen sehen tendenziell eine Zunahme der Kontrollmöglichkeiten der arbeitenden Menschen (Abb. 2.7). Dieser Effekt ist allerdings nur bei den dispositiven Tätigkeiten klar und relativ widerspruchsfrei erkennbar. Für einen gewissen Anteil operativer Tätigkeiten (rund $20 \%$ ) wird auch eine Abnahme der Kontrolle des Menschen über sein Arbeitsumfeld gesehen. Offensichtlich sehen die Autoren hier für die operativen Tätigkeiten eine höhere Gefahr des Kontrollverlusts durch „smarte“ Automatisierung.

In krassem Gegensatz dazu wird hinsichtlich der Selbstbestimmung nur für die operativen Tätigkeiten ein Zuwachs erwartet; hier wird möglichweise für die dispositiven Tätigkeiten ein Deckeneffekt gesehen. Die Selbstbestimmung ist hier bereits sehr weit ausgeprägt und kann sich - gemäß dieser Einschätzung - kaum noch weiter entwickeln. 


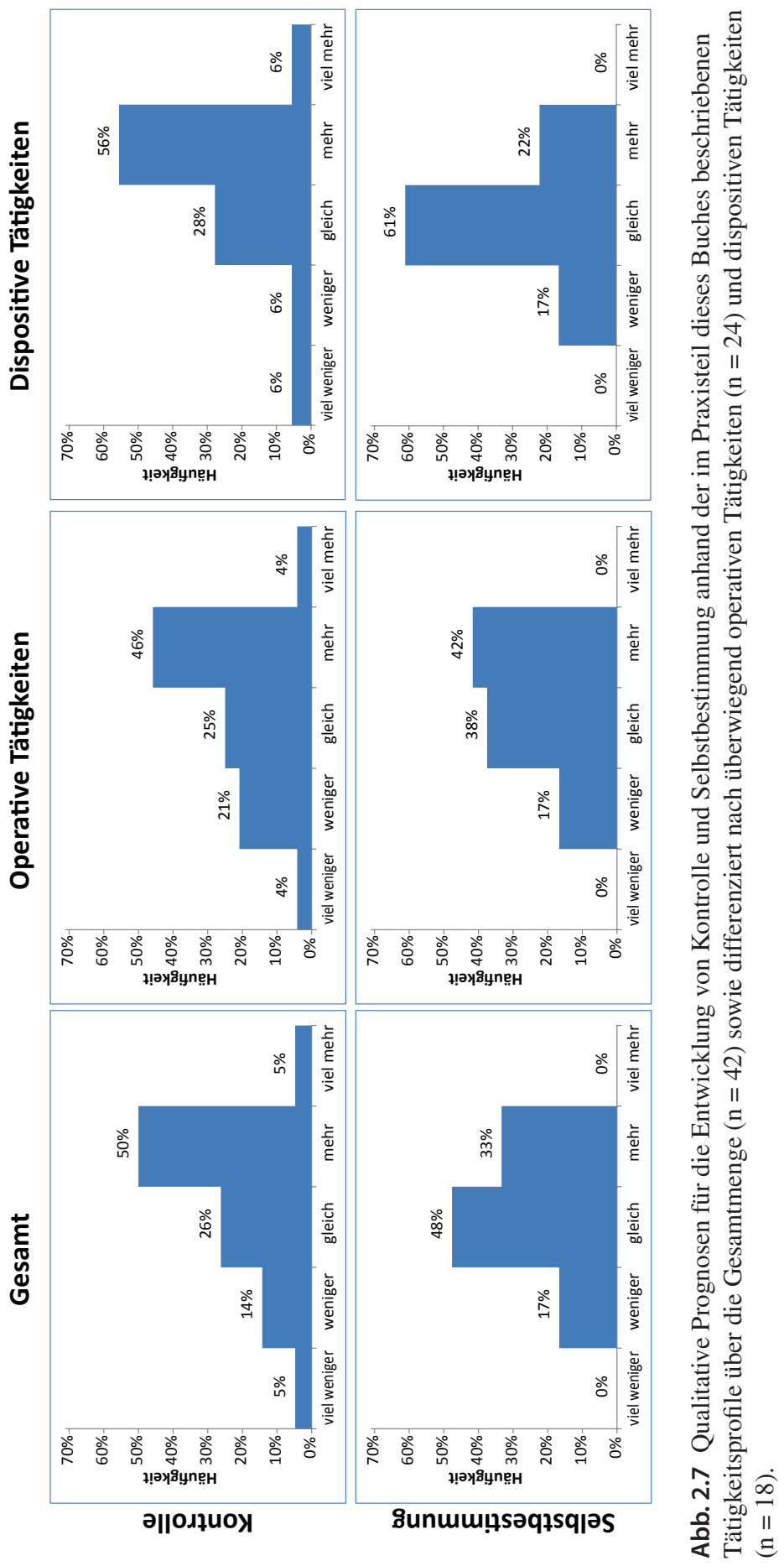




\subsubsection{Querschnittliche und gegenstandsspezifische Aspekte: Interdisziplinarität und IT-Kenntnisse}

Die Dimension der Interdisziplinarität hat einen querschnittlichen Charakter, weil sie sich sowohl auf Kooperationen im Arbeitsumfeld (ein Aspekt der sequentiellen Vollständigkeit) als auch auf Qualifikationserfordernisse (ein Aspekt der hierarchischen Vollständigkeit) bezieht. Im Hinblick auf Kooperationen ist hier die Zusammenarbeit zwischen Fachleuten unterschiedlicher Disziplinen gemeint. Im Hinblick auf Qualifikationserfordernisse geht es um zunehmende Interdisziplinarität in bestimmten Bildungsprofilen, also Interdisziplinarität nicht nur, wie oben angesprochen, zwischen Personen, sondern auch innerhalb einer Person.

Im Kontext der Diskussion um Industrie 4.0 betrifft diese Interdisziplinarität speziell die Integration IT- oder informatikbezogener Kenntnisse mit anderen, bereits etablierten Qualifikationen, insbesondere in der Mechanik und Elektronik. Damit wäre zugleich übergeleitet zur folgenden und letzten Beschreibungsdimension.

Ein Spezifikum der technischen Grundlagen von Industrie 4.0 ist die Digitalisierung, die Integration von informationstechnischen und informatischen Komponenten in mechanische oder mechatronische Systeme. In diesem Sinne sind Anforderungen hinsichtlich neuer und spezifischer IT-Kenntnisse für bestimmte Berufsgruppen in bestimmten Anwendungsfeldern von besonderem Interesse.

Bei der Interdisziplinarität erwarten die Autoren tendenziell Zuwächse, allerdings bei den operativen Tätigkeiten sehr viel weniger deutlich als bei den dispositiven Tätigkeiten (Abb. 2.8). Verwundern könnte, dass für beide Tätigkeitsprofile kein nennenswerter Zuwachs an notwendigen IT-Kenntnissen erwartet wird, obwohl viele Beobachter davon ausgehen, dass die Digitalisierung alle Bereiche des Arbeitslebens durchdringen wird. Anderseits kann die Vereinfachung der Nutzung digitaler Systeme (bspw. durch entsprechende Assistenzsysteme oder vereinfachte Programmierumgebungen) bei deren gleichzeitiger Zunahme erklären, warum in vielen Fällen nicht prognostiziert wird, dass zunehmend ITKenntnisse notwendig sein werden.

\subsection{Gesamtbetrachtung der folgenden Anwendungsbeispiele}

Es sollen nun abschließend die sowohl im Gesamtüberblick als auch in der Detailbetrachtung auffällige Ergebnisse diskutiert werden. Abb. 2.9 fasst die oben im Detail beschriebenen Aspekte in einer Gesamtübersicht zusammen.

Hinsichtlich der hierarchischen Vollständigkeit von Tätigkeiten zeigen sich deutliche Effekte im Sinne einer Abnahme monotoner und einer Zunahme komplexer Aufgaben, auch Optimierung scheint wichtiger zu werden. Hinsichtlich der Problemlösung scheint die Mittelwertsdarstellung in Abb. 2.9 darauf hinzudeuten, dass keinerlei Dynamik zu erwarten ist, Tatsächlich zeigte die differenzierte Betrachtung aber eine ambivalente 


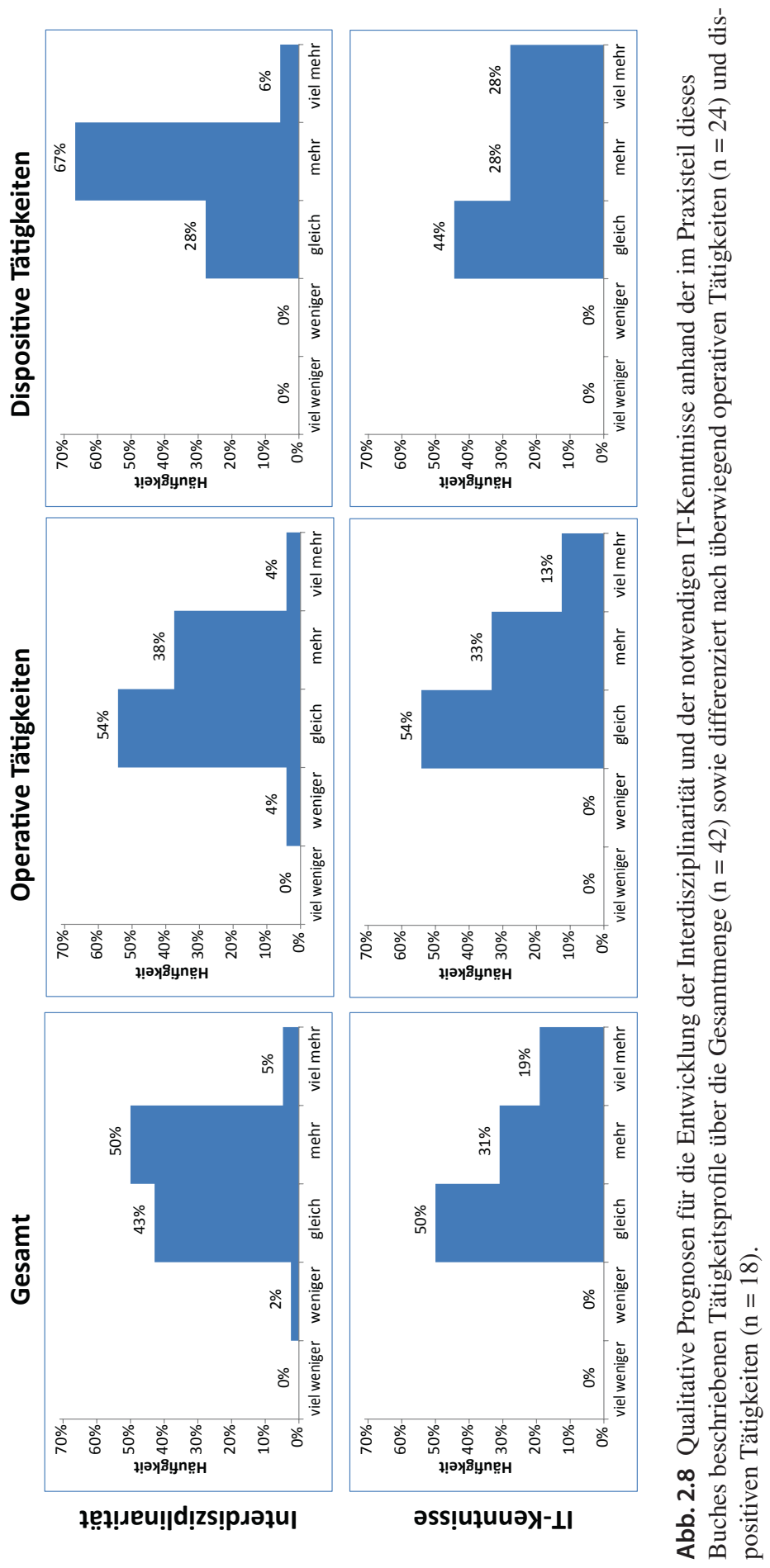




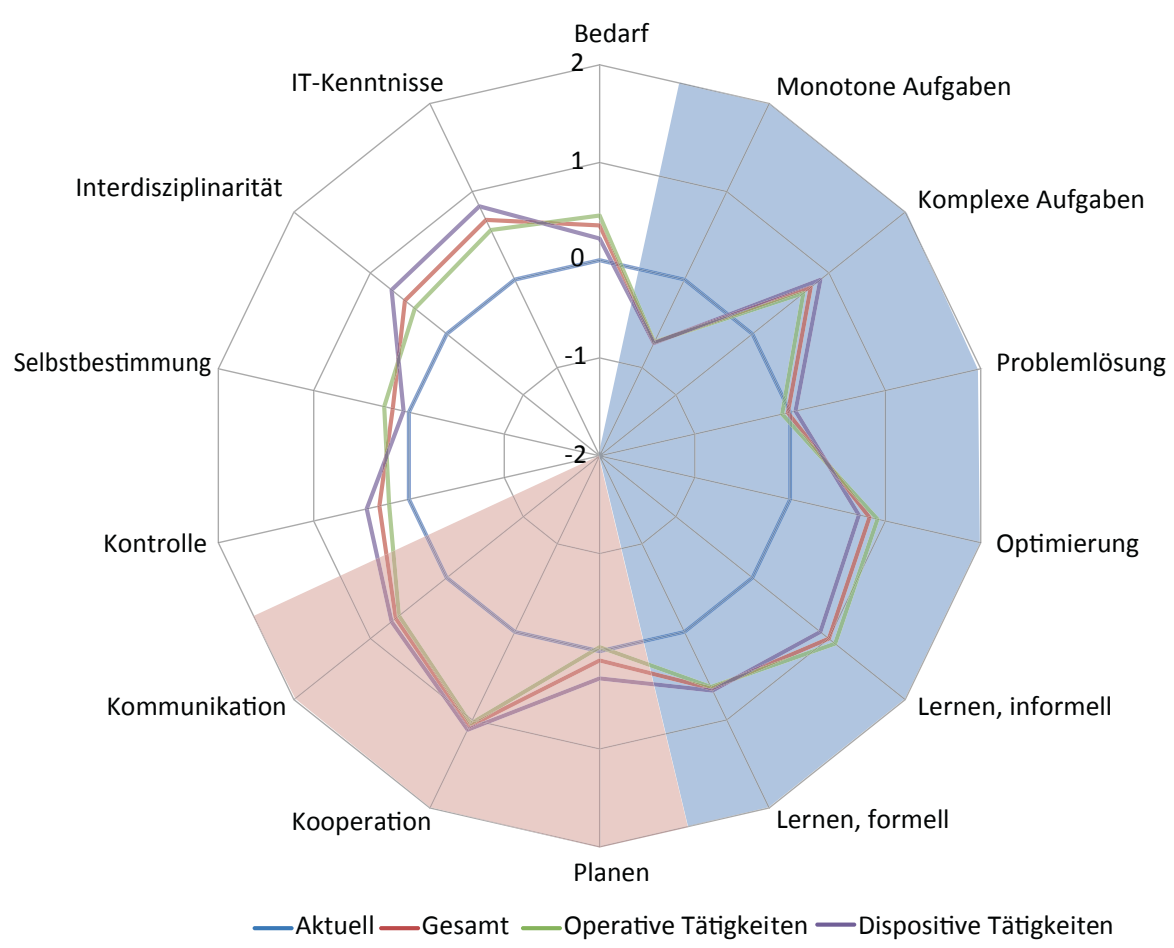

Abb. 2.9 Gesamtüberblick über die prognostizierten Entwicklungen in den unterschiedlichen Arbeitssystemdimensionen. In den blauen Segmenten finden sich wichtige Aspekte der hierarchischen, in den roten Segmenten wichtige Aspekte der sequentiellen Vollständigkeit von Tätigkeiten.

Einschätzung (Abb. 2.3): Sowohl eine Unterstützung wie auch die Ersetzung menschlicher Problemlösung durch „smarte“ Automatisierungstechnik ist denkbar.

Für das Lernen werden insgesamt und in jeder Hinsicht Zuwächse erwartet. Informelles Lernen wird eine deutlich größere Rolle spielen und in qualitativ neuer Weise durch die neuen Technologien unterstützt werden. Formelles Lernen bleibt weiter wichtig und wird sogar an Bedeutung gewinnen, dies sogar auf dispositiver Ebene bei Mitarbeitern, die bereits über hohe Qualifikationen verfügen.

Im Bereich der sequentiellen Vollständigkeit von Tätigkeiten scheint für den Aspekt des Planens erneut die Mittelwertsbetrachtung in Abb. 2.9 wenig Dynamik anzuzeigen. Auch hier wurden in der differenzierten Betrachtung (Abb. 2.5) Nuancen deutlich: Besonders hinsichtlich der operativen Tätigkeitsprofile werden, wie bei der Problemlösung, den Menschen unterstützende Potenziale der Technik ebenso gesehen wie den Menschen ersetzende.

Für Kooperation und Kommunikation (Abb. 2.6) werden hingegen in jeder Hinsicht Zuwächse erwartet.

Wenn auch nuanciert im Detail, so wird doch insgesamt aus den Praxisfällen ein - im Sinne der menschengerechten Arbeit wie auch der Innovationsfähigkeit der Volkswirtschaft - optimistischer Ausblick auf die Zukunft deutlich. 
Dieser Befund sollte mit Vorsicht und Sorgfalt interpretiert werden. Wie bereits eingangs erwähnt, erhebt die hier getroffene Auswahl von Gestaltungsfällen keinerlei Anspruch auf Repräsentativität. Es spricht sogar einiges dafür, dass es sich hier um eine „Positivauswahl“" von Projekten handelt, die besonders gut ausgeprägte Potenziale einer progressiven Arbeitsgestaltung aufweisen.

Nun soll aus vielfältigen Gründen ohnehin nicht der Eindruck eines technologischen Determinismus erweckt werden: Es werden sich aus den technologischen Entwicklungen allein nicht quasi naturgesetzlich bestimmbare Auswirkungen für die Qualität der Arbeit und die Innovationsfähigkeit ergeben. Diese Auswirkungen werden vielmehr abhängen von ganz konkreten unternehmerischen, betrieblichen Entscheidungen, insbesondere im Bereich der Arbeitsorganisation. Was die hier dargestellten Praxisbeispiele leisten, ist - quer durch unterschiedliche Branchen und Anwendungsfelder - der Nachweis der Möglichkeit (nicht der Notwendigkeit) lern- und innovationsförderlicher Arbeitssystemgestaltung.

In den folgenden Kapiteln werden - in diesem Sinne - Anwendungsbeispiele aus aktuellen Forschungsprojekten nach einer gemeinsamen Systematik und im Hinblick auf die hier dargestellten Beschreibungs- und Gestaltungsdimensionen dargestellt. Dadurch besteht die Möglichkeit, einen plastischen Eindruck von Gestaltungspotenzialen und -herausforderungen für die Arbeit in der Industrie $4.0 \mathrm{zu}$ gewinnen.

Dies kann verstanden werden als ein Beitrag zum systematischen Aufbau von Gestaltungswissen in diesem Kontext. Dieser Prozess des Aufbaus von Gestaltungswissen ist erst am Anfang. Die folgenden Beispiele liefern erste Hypothesen für Gestaltungslösungen und -paradigmen, die in weiteren Schritten auf ihre Generalisierbarkeit untersucht werden müssen. Dazu wird es auch notwendig sein, möglichst hypothesen- und theoriegeleitet weitere Gestaltungsfälle aus unterschiedlichen Anwendungskontexten heranzuziehen. Die aktuellen FuE-Programme, etwa die des BMWi und des BMBF, stellen dafür ein fruchtbares Feld dar.

\section{Literatur}

Bainbridge, L. (1983). Ironies of automation. Automatica, 19(6), 775-779. https://doi. org/10.1016/0005-1098(83)90046-8.

Bergmann, B., Fritsch, A., Göpfert, P., Richter, F., Wardanjan, B., \& Wilczek, S. (2000). Kompetenzentwicklung und Berufsarbeit. Münster: Waxmann. https://books.google.de/books?id=JxBrWsvRUIC, zugegriffen am 21.08.2016.

Bergmann, B., Richter, F., Pohlandt, A., Pietrzyk, U., Eisfeld, D., Hermet, V., \& Oschmann, D. (2004). Arbeiten und Lernen. Münster: Waxmann. https://books.google.de/books?id=DQPJqBMJVKoC, zugegriffen am 21.08.2016.

Botthof, A., \& Hartmann, E. A. (Hrsg.) (2015). Zukunft der Arbeit in Industrie 4.0. Berlin Heidelberg: Springer.

Cohen, W. M., \& Levinthal, D. A. (1990). Absorptive capacity. A new perspective on learning and innovation. Administrative Science Quarterly, 35(1), 128. https://doi.org/10.2307/2393553. 
Dengler, K., \& Matthes, B. (2015): Folgen der Digitalisierung für die Arbeitswelt. Substituierbarkeitspotenziale von Berufen in Deutschland. IAB Forschungsbericht (IAB Forschungsbericht, 11/2015). http://doku.iab.de/forschungsbericht/2015/fb1115.pdf, zugegriffen am 21.08.2016.

Erpenbeck, J., Heyse, V., Meynhardt, T., \& Weinberg, J. (2007). Die Kompetenzbiographie. Wege der Kompetenzentwicklung (2., aktualisierte und überarb. Aufl.). Münster: Waxmann. http://www. content-select.com/index.php?id=bib_view\&ean=9783830968085, zugegriffen am 21.08.2016

Frey, C. B., \& Osborne, M. A. (2013). The future of employment: How susceptible are jobs to computerisation?. Oxford: Oxford Martin School, University of Oxford.

Frieling, E., Bernard, H., Bigalk, D., \& Müller, R. F. (2006). Lernen durch Arbeit. Entwicklung eines Verfahrens zur Bestimmung der Lernmöglichkeiten am Arbeitsplatz. Münster: Waxmann.

Grote, G. (1997). Autonomie und Kontrolle. Zur Gestaltung automatisierter und risikoreicher Systeme. Zürich: vdf Hochschulverl. an der ETH Zürich (Mensch, Technik, Organisation, 16).

Hacker, W. (1995). Tätigkeitsbewertungssystem (TBS); Verfahren zur Analyse, Bewertung und Gestaltung von Arbeitstätigkeiten. Zürich: vdf Hochschulverl. (Mensch, Technik, Organisation, 7).

Hacker, W. (2005). Allgemeine Arbeitspsychologie. Psychische Regulation von Wissens-, Denk- und körperlicher Arbeit (2., vollst. überarb. und erg. Aufl.). Bern: Huber (Schriften zur Arbeitspsychologie, 58).

Hacker, W., \& Richter, P. (1990). Psychische Regulation von Arbeitstätigkeiten - Ein Konzept in Entwicklung. In: F. Frei \& I. Udris (Hrsg.), Das Bild der Arbeit. Bern: Huber.

Hartmann, E. A. (2005). Arbeitssysteme und Arbeitsprozesse. Zürich: vdf Hochschulverlag.

Hartmann, E. A., \& Bovenschulte, M. (2013). Skills needs analysis for "Industry 4.0" based on roadmaps for smart systems. In: Using technology foresights for identifying future skills needs. Global Workshop Proceedings. Moskow. Skolkovo, International Labour Organization.

Hartmann, E. A., von Engelhardt, S., Hering, M., Wangler, L., \& Birner, N. (2014). Der iit-Innovationsfähigkeitsindikator. Ein neuer Blick auf die Voraussetzungen von Innovationen. Berlin: iit. http://www.iit-berlin.de/de/publikationen/der-iit-innovationsfaehigkeitsindikator/, zugegriffen am 21.08.2016

Hartmann, E. A., \& Garibaldo, F. (2011). What's going on out there? Designing work systems for learning in real life. In: S. Jeschke, I. Isenhardt, F. Hees, \& S. Trantow (Hrsg.), Enabling innovation: Innovative capability - German and international views. Berlin: Springer Berlin.

Henning, K., Volkholz, V., Risch, W., \& Hacker, W. (Hrsg.) (1994). Moderne LernZeiten. Berlin/ Heidelberg/New York: Springer.

Hirsch-Kreinsen, H. (2015). Entwicklungsperspektiven von Produktionsarbeit. In: A. Botthof \& E. A. Hartmann (Hrsg.), Zukunft der Arbeit in Industrie 4.0. Berlin Heidelberg: Springer.

Hirsch-Kreinsen, H., Ittermann, P., \& Niehaus, J. (Hrsg.) (2015). Digitalisierung industrieller Arbeit. Die Vision Industrie 4.0 und ihre sozialen Herausforderungen. Baden-Baden: Nomos/ Edition Sigma.

Kauffeld, S. (2007). Jammern oder Lösungsexploration? Eine sequenzanalytische Betrachtung des Interaktionsprozesses in betrieblichen Gruppen bei der Bewältigung von Optimierungsaufgaben. Zeitschrift für Arbeits- und Organisationspsychologie A\&O, 51(2), 55-67. https://doi. org/10.1026/0932-4089.51.2.55.

Lorenz, E. (2015). Work organisation, forms of employee learning and labour market structure. Accounting for international differences in workplace innovation. Journal of the Knowledge Economy, 6(2), 437-466. https://doi.org/10.1007/s13132-014-0233-4.

Lorenz, E., \& Valeyre, A. (2005). Organisational innovation, human resource management and labour market structure. A comparison of the EU-15. Journal of Industrial Relations, 47(4), 424-442. https://doi.org/10.1111/j.1472-9296.2005.00183.x.

Lüdtke, A. (2015). Wege aus der Ironie in Richtung ernsthafter Automatisierung. In: A. Botthof \& E. A. Hartmann (Hrsg.), Zukunft der Arbeit in Industrie 4.0 (S. 125-146). Berlin Heidelberg: Springer. 
Mühlbradt, T. (2015). Was macht Arbeit lernförderlich? Eine Bestandsaufnahme. Stuttgart: MTMInstitut (MTM-Schriften Industrial Engineering, Ausg. 1).

OECD. (2010). Recognising non-formal and informal learning. Paris: OECD Publishing.

Oesterreich, R., \& Dunckel, H. (1993). Kontrastive Aufgabenanalyse im Büro. Der KABA-Leitfaden. Zürich: vdf Verl. d. Fachvereine (Mensch, Technik, Organisation, 5).

Pietzcker, F. (Hrsg.) (2010). Der Aufgabenbezogene Informationsaustausch. Zeitweilige partizipative Gruppenarbeit zur Problemlösung; mit besonderem Blick auf Organisationsentwicklung, Wissensmanagement und betriebliche Gesundheitsvorsorge. Zürich: vdf Hochschulverl. (Mensch - Technik - Organisation, 45).

Richter, P., \& Hacker, W. (1997). Psychische Fehlbeanspruchung. Heidelberg: Asanger.

Seligman, M. E. P. (1995). Erlernte Hilflosigkeit (5., korrigierte Aufl., erw. um: Franz Petermann: Neue Konzepte). Weinheim: Beltz Psychologie-Verl.-Union.

Sell, R., \& Schimweg, R. (2002). Probleme lösen. In komplexen Zusammenhängen denken (mit 19 Tabellen. 6., korrigierte Aufl.). Berlin: Springer (Engineering online library).

Stewart, I., Debapratim, D., \& Cole, A. (2015): Technology and people: The great job-creating machine. London: Deloitte LLP. http://www2.deloitte.com/content/dam/Deloitte/uk/Documents/finance/deloitte-uk-technology-and-people.pdf, zugegriffen am 21.08.2016.

Ulich, E. (1978). Über das Prinzip der differentiellen Arbeitsgestaltung. Industrielle Organisation, 47, 566-568.

Ulich, E. (2005). Arbeitspsychologie (6., überarb. und erw. Aufl.). Zürich: vdf Hochschulverl. an der ETH. http://deposit.ddb.de/cgi-bin/dokserv?id=2646503\&prov=M\&dok_var=1\&dok_ ext=htm, zugegriffen am 21.08.2016.

Ulich, E. (2016). Differenzielle Arbeitsgestaltung - Ein zukunftsfähiges Konzept. In: Institut für Arbeitsforschung und Organisationsberatung (iafob) (Hrsg.), Unternehmensgestaltung im Spannungsfeld von Stabilität und Wandel. Band 2: Neue Erfahrungen und Erkenntnisse. Zürich: vdf Hochsch.-Verl. an der ETH (Mensch - Technik - Organisation, 43).

Weber, W. G. (1997). Analyse von Gruppenarbeit. Kollektive Handlungsregulation in soziotechnischen Systemen (Eidgenössische Techn. Hochsch., Habil.-Schr.--Zürich, 1996, 1. Aufl.). Bern: Huber (Schriften zur Arbeitspsychologie, 57).

Zölch, M., Weber, W. G., \& Leder, L. (Hrsg.) (1999). Praxis und Gestaltung kooperativer Arbeit. Zürich: vdf Hochschulverl. an der ETH Zürich (Mensch, Technik, Organisation, 23).

Open Access Dieses Kapitel wird unter der Creative Commons Namensnennung 4.0 International Lizenz (http://creativecommons.org/licenses/by/4.0/deed.de) veröffentlicht, welche die Nutzung, Vervielfältigung, Bearbeitung, Verbreitung und Wiedergabe in jeglichem Medium und Format erlaubt, sofern Sie den/die ursprünglichen Autor(en) und die Quelle ordnungsge-mäß nennen, einen Link zur Creative Commons Lizenz beifügen und angeben, ob Änderungen vorgenommen wurden.

Die in diesem Kapitel enthaltenen Bilder und sonstiges Drittmaterial unterliegen ebenfalls der genannten Creative Commons Lizenz, sofern sich aus der Abbildungslegende nichts anderes ergibt. Sofern das betreffende Material nicht unter der genannten Creative Commons Lizenz steht und die betreffende Handlung nicht nach gesetzlichen Vorschriften erlaubt ist, ist für die oben aufgeführten Weiterverwendungen des Materials die Einwilligung des jeweiligen Recht-einhabers einzuholen.

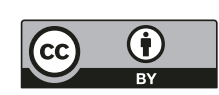

